\title{
(2) OPEN ACCESS \\ The dynamics in applied COVID-19 pharmacotherapy and the influence of national guidance in The Netherlands: a quantitative and qualitative study
}

\author{
Elien B Uitvlugt $\left({ }^{0},{ }^{1}\right.$ Danny Y F Soeng, ${ }^{2}$ Paul van der Linden, ${ }^{3}$ \\ Ewoudt M W van de Garde (i) 2,4
}

- Additional supplemental material is published online only. To view, please visit the journal online (http://dx. doi.org/10.1136/ejhpharm2021-002781)

'Hospital Pharmacy, OLVG, Amsterdam, The Netherlands ${ }^{2}$ Clinical Pharmacy, Sint Antonius Hospital, Nieuwegein, The Netherlands

${ }^{3}$ Clinical Pharmacy, Tergooi, Hilversum, The Netherlands

${ }^{4}$ Division of

Pharmacoepidemiology and Clinical Pharmacology, Utrecht University, Utrecht, Netherlands

Correspondence to Elien B Uitvlugt, OLVG, Amsterdam, The Netherlands; e.uitvlugt@olvg.nl

Received 5 March 2021 Accepted 8 June 2021

EAHP Statement 5: Patient Safety and Quality Assurance.

Check for updates

(C) European Association of Hospital Pharmacists 2021. Re-use permitted under CC BY-NC. No commercial re-use. Published by BMJ.

\begin{tabular}{l}
\hline To cite: Uitvlugt EB, \\
Soeng DYF, van der Linden P, \\
et al. Eur J Hosp Pharm Epub \\
ahead of print: [please \\
include Day Month Year]. \\
doi:10.1136/ \\
ejhpharm-2021-002781
\end{tabular}

\section{ABSTRACT \\ Objective At the beginning of the COVID-19} pandemic in the Netherlands, the Dutch Working Party on Antibiotic Policy constructed an advisory document about off-label drug treatment options that was regularly updated with new scientific findings. The aim of this study is to describe the dynamics in applied COVID-19 pharmacotherapy during the first 100 days of the pandemic and to assess how the national advisory document influenced local hospital policies.

Methods A multicentre observational cohort study was conducted in six hospitals in the Netherlands. Patients with confirmed COVID-19 admitted between 27 February and 7 June 2020 were studied. Drug prescription data were collected and percentages of patients receiving a specific treatment were calculated. These percentages were plotted together with release dates of the national advisory document. Semi-structured in-depth interviews with hospital pharmacists and infectious diseases specialists were conducted to gain insight into the development and implementation of pharmacotherapy treatment protocols in hospitals.

Results Data from 1511 patients (60\% men, mean age 66 years) were analysed. From mid-March (hydroxy) chloroquine was being prescribed in all six hospitals to approximately $70 \%$ of patients at admission. Frequencies of other off-label treatments were below $2 \%$. In the week of 6 April 2020, the first hospital discontinued prescribing (hydroxy)chloroquine and the last hospital discontinued in the week of 4 May 2020 (total range -19 to +10 days after the national advisory document advised against its use (1 May 2020)). All interviewees $(n=6)$ stated that the hospitals based their policies mainly on the national advisory document but also assessed scientific literature themselves. Order panels were constructed to support prescribing.

Conclusion Dutch hospitals opted en masse for (hydroxy)chloroquine as COVID-19 therapy at the start of the pandemic, although the time until the therapy was no longer prescribed differed by several weeks. The fact that hospitals defined pharmacotherapy regimens based on their own assessment of the scientific literature besides the national advisory document can explain this variation.

\section{INTRODUCTION}

Since the beginning of 2020 the world has been facing the enormous impact of coronavirus disease 2019 (COVID-19). Especially during the first months of the pandemic, there was much uncertainty regarding the optimal pharmacotherapy, which led to the frequent use of off-label therapies in many countries. ${ }^{12}$ In the Netherlands, the Dutch Working Party on Antibiotic Policy (SWAB) composes evidence-based guidelines regarding optimal utilisation of antibiotics with the aim of limiting the development of antimicrobial resistance. On 18 March 2020, SWAB released a national advisory document which summarised evidence and provided advice on the use and non-use of pharmacotherapeutic options against COVID-19 for the purpose of supporting Dutch hospitals with keeping on track with rapidly increasing scientific knowledge. ${ }^{3}$ This document has been regularly updated following its first release. However, it is unknown how this document influenced local hospital pharmacotherapeutic policies.

The aim of this study is therefore to describe the dynamics in applied COVID-19 pharmacotherapy in the Netherlands and to assess how the national advisory document influences local hospital policies.

\section{METHODS}

\section{Study design and setting}

Six hospitals geographically spread across the Netherlands participated in the study (five large teaching hospitals from the Santeon network (www.santeon. nl) and one general hospital (Tergooi Hospital)). Hospitals identified all patients (aged $>18$ years) with confirmed COVID-19 admitted to a non-ICU ward between 27 February and 7 June 2020 and not discharged, transferred to another hospital or ICU ward, or died within 24 hours from admission. Next, for all these patients, drug prescription data from the day of admission were extracted from the Santeon Farmadatabase ${ }^{4}$ for all hospitals except one, which extracted data from the local Electronic Health Record system directly. Based on these prescription data, percentages of patients receiving a specific treatment were calculated in 7-day intervals. Subsequently, these percentages were plotted together with release dates of the aforementioned national advisory document. The advice from this document is summarised in online supplemental figure 1. Besides the quantitative assessment of prescription practice, all hospitals were invited to participate in an interview study. From each participating hospital, one infectious disease specialist or hospital pharmacist involved in the development of local treatment protocols for COVID-19 was interviewed. Interviews lasted $30 \mathrm{~min}$ and were conducted by video conference. A semi-structured 
questionnaire was used consisting of three main topics: development, implementation and evaluation of treatment protocols (see online supplemental interview guide). All interviews were recorded and transcribed verbatim. The transcripts were sent to the interviewees for assessing completeness and accurateness.

\section{Data analyses}

All data were analysed in Microsoft Excel 2016 (Microsoft, Redmond, Washington, USA). Descriptive statistics were used to describe patients' characteristics and pharmacotherapy regimen differences in type of antiviral, anti-inflammatory and antimicrobial drugs prescribed at day of admission in the different hospitals.

All six transcripts were analysed by directed content analysis, a systematic process in which codes are assigned to specific text portions and thereafter classified based on an existing theory. ${ }^{5}$ In this study, the theory of Fleuren et al was used. They identified groups of determinants that may affect successful implementation of healthcare innovations such as guidelines. ${ }^{6}$

Codes were assigned using ATLAS.ti version 9.0.15 (ATLAS. ti Scientific Software Development GmBH, Berlin, Germany). The transcripts were coded by a single person (DYFS) followed by a second round of coding by another person (EBU). Subsequently, all codes were compared and discussed until consensus was achieved. Afterwards, all codes were grouped according to the theory of Fleuren et al into three categories: the guideline, the organisation and the users.

\section{RESULTS}

\section{Patient selection and baseline characteristics}

In total, 1812 patients with confirmed COVID-19 were hospitalised in the time frame under study, of which 174 (9.6\%) were admitted to the ICU within 24 hours after presentation at the emergency department, $14(0.8 \%)$ died within 24 hours, 78 $(4.3 \%)$ were discharged home, $27(1.5 \%)$ were transferred to another hospital and $8(0.4 \%)$ were transferred to a location elsewhere. Hence, 1511 patients remained for the quantitative analysis, with $905(59.9 \%)$ men and with a mean age of 66.0 years.

\section{Prescribed drugs}

During the period under study, the following drugs discussed in the national advisory document were prescribed to one or more patients: (hydroxy)chloroquine $(n=885)$, azithromycin $(n=24)$, lopinavir/ritonavir $(n=6)$, oseltamivir $(n=4)$. Because (hydroxy) chloroquine appeared the only drug to be prescribed extensively, further analyses focused solely on (hydroxy)chloroquine (dosage regimen: hydroxychloroquine day 1: $400 \mathrm{mg}$ twice a day and days 2-5: $200 \mathrm{mg}$ twice a day; chloroquine: day 1: start $600 \mathrm{mg}$,

Edition of the national advisory document for COVID-19 of the Duth working party on antibiotic policy:

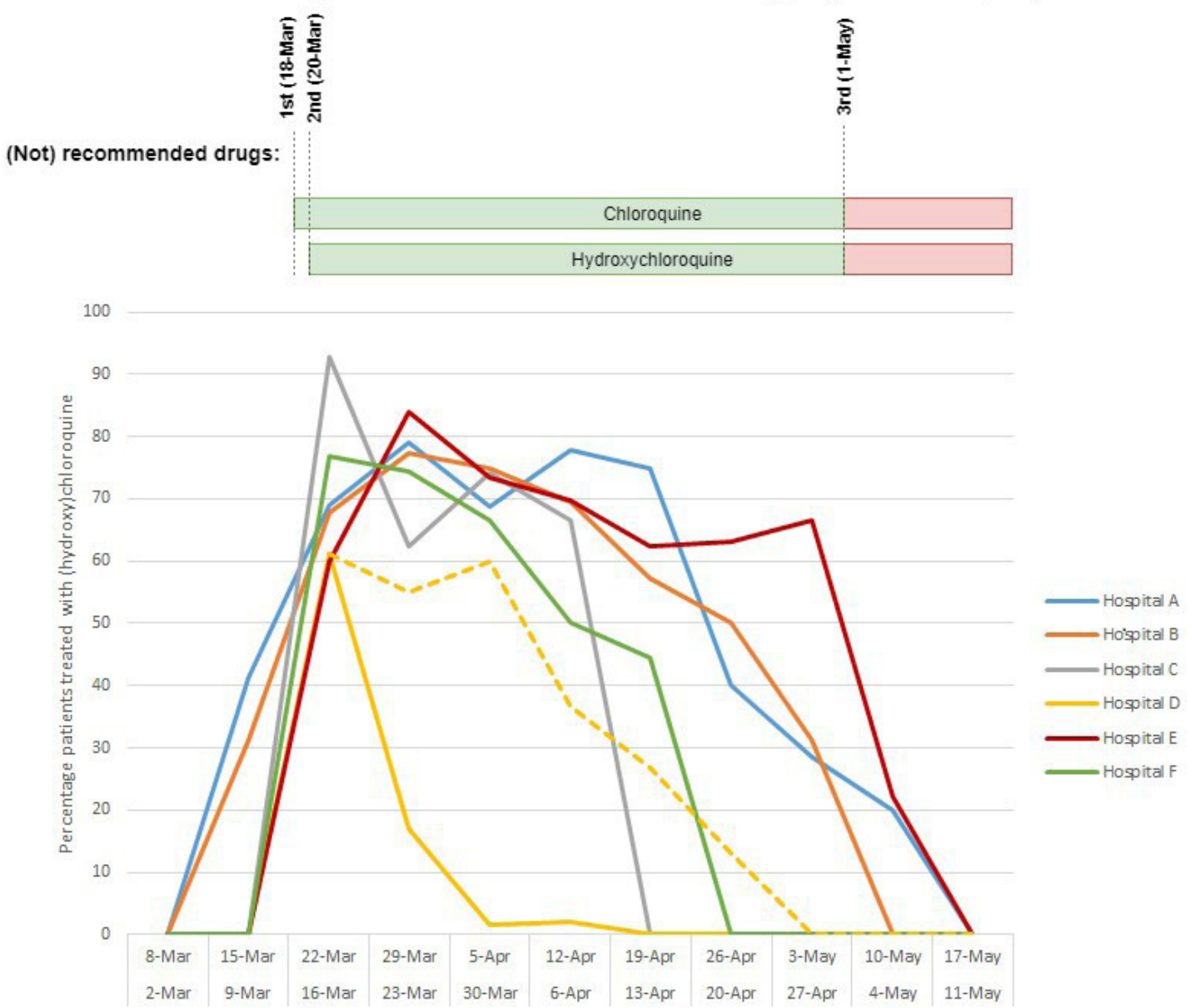

Figure 1 Weekly percentage of patients treated with (hydroxy)chloroquine against the time of the study within which the advice was valid. Weeks with $<5$ hospitalisations were ignored. Continuous line shows chloroquine, dotted line shows hydroxychloroquine. 
following $300 \mathrm{mg}$ after 12 hours and days 2-5: $300 \mathrm{mg}$ twice a day)

The percentage of patients treated with (hydroxy)chloroquine per hospital per week is shown in figure 1. In the week of 2 March 2020, the first hospitals started with (hydroxy)chloroquine treatment, whereas other hospitals followed 1 week later when the national advisory document was released. On average, $70 \%$ of patients admitted were prescribed (hydroxy)chloroquine until the first hospital discontinued treatment in the week of 6 April 2020. No patients were prescribed (hydroxy)chloroquine after 11 May 2020. The national advisory document advised against its use from 1 May 2020 because there was insufficient evidence of the effectiveness of (hydroxy)chloroquine treatment for patients with COVID-19.

\section{Local treatment policies}

In total, four hospital pharmacists and two infectious disease specialists involved in hospital policy-making of the treatment of COVID-19 were interviewed. Their responses are shown in online supplemental table 1 . In summary, all hospitals mainly based their policies on the national advisory document. One hospital used this document unchanged, while other hospitals made hospital-specific adjustments to fit the local clinical setting. Besides the national advisory document, hospitals also assessed scientific literature due to rapidly evolving scientific insights.

In all hospitals, already existing or newly formed multidisciplinary teams were responsible for the development and implementation of hospital policies. These teams consisted of at least hospital pharmacists, infection disease specialists and pulmonologists. Decisions were made rapidly and easily in frequent (ad hoc) meetings, because all healthcare was focused on COVID-19.

During the implementation phase, order sets were developed in the electronic patient record to ease the prescription of COVID-19 medication and changes were actively communicated to the users of the policies. Due to the COVID-19 focus of all healthcare providers, the policies were widely supported. Deviations from hospital policies did consciously occur due to clinical assessment of patient characteristics and co-medication.

\section{DISCUSSION}

This study shows that (hydroxy)chloroquine treatment was prescribed to the majority of patients in all hospitals from the start of the pandemic. In contrast to the adoption of (hydroxy) chloroquine treatment, the timing of discontinuing prescribing (hydroxy)chloroquine showed more variation $(-19$ to +10 days after the national advisory document advised against its use).

The fact that hospitals determined pharmacotherapy regimens based on their own assessment of the scientific literature besides the national advisory document can explain this variety in discontinuation.

Although the interviews yielded positive opinions about the availability of the national advisory document, several suggestions for improvement could be identified: (1) addition of advice regarding specific patient populations (such as impaired kidney function and dialysis); (2) consideration of medicinal shortages and costs; and (3) evaluation of the medicinal safety of off-label treatments in association with the Dutch pharmacovigilance centre Lareb.

Possible improvements concerning the hospital are prevention of tension and incomprehension between wards by harmonising tasks and expectations, harmonisation of regional COVID-19 approach due to patient transfers between hospitals and communication of adjustments of hospital policies to all users including non-prescribers as users who are responsible for updating order sets parallel to policy adjustments.

Because of the design of the study, collecting and analysing prescription data parallel with the execution of the interviews, a limitation of the study is that we were not able to discuss the prescription data during the interviews. This prevents conclusions about causal relations between approaches to local policy making and prescription practices. Furthermore, we were not able to assess whether alterations in prescribing practices were solely linked to modifications in policy or influenced by suboptimal implementation as well. Finally, as this study was performed in the Dutch context, this potentially limits the generalisability to other nations around the globe.

The results of this study provide a relevant example of how to assess the interplay between a dynamic national advisory document and local treatment policy making under exceptional circumstances. We think that these types of studies can help to identify leads to further enhance implementation practice of guidelines that tend to have a dynamic character. Extending the initially determined study period up to the present time could provide additional insights into the evolution of pharmacotherapy prescribing practice in a context of ongoing scientific research on the optimal treatment of COVID-19.

\section{CONCLUSION}

This study shows that, in the Netherlands, hospitals opted en masse for (hydroxy)chloroquine as COVID-19 therapy during the first 100 days of the pandemic, although the time until the treatment was no longer prescribed differed substantially. The fact that hospitals determined pharmacotherapy regimens based on their own assessment of the scientific literature, besides the national advisory document, can explain this variation. The in-hospital interviews which provided more emphasis on drug safety as well as costs in national advisory documents, together with very proactive in-hospital communication about modifications in treatment policies, could be helpful in achieving timely best evidence-based practice.

Correction notice This article has been corrected since it was published online. The last author's middle names have been initialised.

Acknowledgements We would like to thank all the responders to the interviews who have provided details of their institutions: Dr J E Stalenhoef, OLVG Hospital, H W H A Fleuren, Canisius-Wilhelmina Hospital, C H M Kerskes, Catharina Hospital, E T Sportel, Medisch Spectrum Twente, and Dr P J de Vries, Tergooi Hospital. Furthermore, we would like to thank Dr H A J M de Wit, Canisius-Wilhelmina Hospital, J B Masselink, Medisch Spectrum Twente and Dr R ten Broeke, Catharina Hospital for their efforts to complete and validate the hospital data.

Contributors EBU and EMWvdG designed the study. Data collection was performed by EBU. The interviews were conducted by DYFS. EBU and DYFS analysed and interpreted the data. DYFS wrote the initial draft of the manuscript draft. EBU, PvdL and EMWvdG revised and edited the manuscript. The final manuscript was approved by all authors.

Funding This study was supported by the Dutch Working Party on Antibiotic Policy (SWAB) and funded by the Netherlands Organisation for Health Research and Development (ZonMw; project no 10430042010048).

Competing interests None declared.

\section{Patient consent for publication Not required.}

Ethics approval The study was approved by the Santeon Review Board (SDB 2020-003). The need for informed consent was waived because of the retrospective nature of the study and anonymous handling of data.

Provenance and peer review Not commissioned; externally peer reviewed. Data availability statement Data are available upon reasonable request.

Supplemental material This content has been supplied by the author(s). It has not been vetted by BMJ Publishing Group Limited (BMJ) and may not have been peer-reviewed. Any opinions or recommendations discussed are solely those of the author(s) and are not endorsed by BMJ. BMJ disclaims all liability and responsibility arising from any reliance placed on the content. Where the content includes any translated material, BMJ does not warrant the accuracy and reliability 
of the translations (including but not limited to local regulations, clinical guidelines, terminology, drug names and drug dosages), and is not responsible for any error and/or omissions arising from translation and adaptation or otherwise.

Open access This is an open access article distributed in accordance with the Creative Commons Attribution Non Commercial (CC BY-NC 4.0) license, which permits others to distribute, remix, adapt, build upon this work non-commercially, and license their derivative works on different terms, provided the original work is properly cited, an indication of whether changes were made, and the use is noncommercial. See: http://creativecommons.org/licenses/by-nc/4.0/.

\section{ORCID iDs}

Elien B Uitvlugt http://orcid.org/0000-0002-0349-4873

Ewoudt M W van de Garde http://orcid.org/0000-0002-1334-2144

\section{REFERENCES}

1 Kalil AC. Treating COVID-19-off-label drug use, compassionate use, and randomized clinical trials during pandemics. JAMA 2020;323:1897-8.
2 Alvarez A, Cabia L, Trigo C, et al. Prescription profile in patients with SARS-CoV-2 infection hospitalised in Aragon, Spain. Eur J Hosp Pharm 2020. doi:10.1136/ ejhpharm-2020-002476. [Epub ahead of print: 20 Nov 2020].

3 Dutch Working Party on Antibiotic Policy. Medicinal treatment of COVID-19 patients. Dutch Working Party on Antibiotic Policy, 2020. Available: https://swab.nl/nl/covid-19 [Accessed 11 Dec 2020].

4 van de Garde EMW, Plouvier BC, Fleuren HWHA, et al. Pharmacotherapy within a learning healthcare system: rationale for the Dutch Santeon Farmadatabase. Eur J Hosp Pharm 2019;26:46-50.

5 Hsieh H-F, Shannon SE. Three approaches to qualitative content analysis. Qual Health Res 2005;15:1277-88.

6 Fleuren MAH, Paulussen TGWM, Van Dommelen P, et al. Towards a measurement instrument for determinants of innovations. Int I Qual Health Care 2014;26:501-10.

7 Vollaard A, Gieling EM, van der Linden PD, et al. [Hydroxychloroquine and chloroquine for COVID-19: no evidence of effectiveness]. Ned Tijdschr Geneeskd 2020;164:D5141. 